\title{
Vitamin D supply in patients with rheumatic diseases in Poland - a pilot study
}

\author{
Marta Runowska $^{1}$ ID , Dominik Majewski $^{1}$ ID , Katarzyna Majewska² $^{\text {ID }}$, Mariusz Puszczewicz ${ }^{1}$ ID \\ ${ }^{1}$ Department of Rheumatology, Rehabilitation and Internal Medicine, Poznan University of Medical Sciences, Poland \\ ${ }^{2}$ Department of Clinical Auxology and Pediatric Nursing, Poznan University of Medical Sciences, Poland
}

\begin{abstract}
Objectives: In rheumatic diseases, vitamin D supply is recommended as part of the prophylaxis and treatment of osteoporosis, especially in patients undergoing glucocorticoid therapy, but also due to its immunoregulatory and anti-inflammatory properties. We aimed to evaluate serum 25 -hydroxyvitamin $\mathrm{D}\left[25(\mathrm{OH}) \mathrm{D}_{3}\right]$ levels in Polish patients with systemic sclerosis $(\mathrm{SSc})$, systemic lupus erythematosus (SLE) and granulomatosis with polyangiitis (GPA), in relation to various clinical parameters, and to assess the initial range of doses for the purpose of further research.

Material and methods: 112 patients (39 with SLE, 44 with SSC and 29 with GPA), referred to the Department of Rheumatology and Internal Medicine in Poznan, Poland, were enrolled in this retrospective study. Demographic and clinical data were collected, including $25(\mathrm{OH}) \mathrm{D}_{3}$ serum levels, vitamin D supplementation doses and season of blood sampling.

Results: Mean (SD) serum 25(OH)D $\mathrm{D}_{3}$ concentrations were 31 (19.4) ng/ml for SLE, 28.8 (12.5) ng/ml for SSc and 28 (15.2) ng/ml for GPA, and they did not significantly differ between the groups. Vitamin D levels below the optimal range were found in $43.8 \%$ of SLE, $65.9 \%$ of SSC and $72.4 \%$ of GPA patients. $80 \%$ of patients reported vitamin D intake, with a mean daily dose of 1398 IU for SLE, 1345 IU for SSc and 1689 IU for GPA. Vitamin D insufficiency and deficiency were frequent among patients with rheumatic diseases, independently of the diagnosis and season.

Conclusions: Patients with rheumatic diseases seem to require higher doses of vitamin $D$ than recommended for the general population. The present results indicate the necessity to use higher initial doses of vitamin D in this group of patients (2000 to $4000 \mathrm{UI}$ ) and to maintain the dose of vitamin $D$ regardless of the change of seasons.
\end{abstract}

Key words: systemic sclerosis, systemic lupus erythematosus, granulomatosis with polyangiitis, hypovitaminosis $\mathrm{D}$.

\section{Introduction}

The classical role of vitamin D in bone metabolism regulation is broadly known [1]. However, after the discovery that vitamin D receptors (VDR) are expressed by several different cells and tissues, not necessarily involved in calcium-phosphate homeostasis [2], scientific attention turned toward the non-classical, extraskeletal properties of vitamin $D$ and its potential pleiotropic effect on human health $[3,4]$.

In recent decades, low vitamin $D$ levels have been associated with increased incidence of several diseases such as cardiovascular diseases [5], diabetes [6], cancers [7], infectious diseases [8] and autoimmune diseases [9]. However, whether vitamin D deficiency plays a causative role, or is rather a marker of poor health status related to chronic disease, remains unclear, as the majority of the studies concerning these associations are observational in nature, whereas evidence from large-scale clinical trials is lacking [5, 10, 11].

In rheumatic diseases, vitamin D supplementation is recommended primarily in the prevention and management of osteoporosis, especially in patients receiving glucocorticoid therapy $[12,13]$.

\section{Address for correspondence:}

Marta Runowska, Department of Rheumatology, Rehabilitation and Internal Medicine, Poznan University of Medical Sciences, 10 Fredry St. 61-701 Poznań, Poland, e-mail: marta.runowska1@gmail.com

Submitted: 29.01.2021; Accepted: 08.06.2021 
Nevertheless, due to its immunomodulatory and anti-inflammatory properties, the potential impact of vitamin D on the prevalence and outcomes in rheumatic diseases has been recently investigated $[9,11]$. For example, numerous studies have demonstrated vitamin D deficiency in patients with systemic lupus erythematosus (SLE) and rheumatoid arthritis (RA) [14-16], as compared to healthy controls. Moreover, vitamin D concentrations have also been negatively correlated with disease activity/severity in both SLE and RA [17-20]. However, further studies are necessary to confirm such a relationship [1].

In the literature, decreased vitamin D levels have also been frequently observed in systemic sclerosis (SSc) [21-23], and several associations have been reported, for instance with disease subtype [21], with specific organ involvement, such as lungs, peripheral vessels or kidneys [24], with digital ulcerations [25] or with extent of skin involvement measured by the Rodnan skin score [23].

Regarding antineutrophil cytoplasmic antibody (ANCA) associated vasculitides (AAV), Perez et al. [26] noted the influence of lower vitamin $D$ serum levels on the increased risk of respiratory infections in patients with GPA. No relationship, though, between $25(\mathrm{OH}) \mathrm{D}_{3}$ concentrations and disease activity has been found, but further investigation is needed to determine the prevalence and significance of hypovitaminosis D in AAV.

The aim of this study was to perform an initial evaluation of the vitamin D supply and serum levels among Polish patients with selected rheumatic diseases, i.e. SSc, SLE and GPA, in relation to various clinical features and glucocorticosteroid therapy, and to estimate the initial range of vitamin $D$ doses for the purpose of further research.

\section{Material and methods}

A cohort of 112 (39 with SLE, 44 with SSc and 29 with GPA) Caucasian patients, referred to the Department of Rheumatology and Internal Medicine, Poznan University of Medical Sciences, Poland, between October 2017 and July 2019, was enrolled in this retrospective study. We accessed the patient records in September 2019.

All patients fulfilled the adequate disease classification criteria: the revised American College of Rheumatology (ACR) criteria for classification of SLE [27]; the ACR/ European League Against Rheumatism (EULAR) 2013 criteria for classification of SSc [28], or the 1990 ACR criteria for the classification of GPA [29], respectively.

Demographic and clinical data were collected, including gender, age, vitamin D and calcium supplementation status, current glucocorticosteroid use, and comorbidities such as osteoporosis, osteoporotic fractures and chronic kidney disease. Serum concentrations of 25-hydroxyvitamin $\mathrm{D}\left[25(\mathrm{OH}) \mathrm{D}_{3}\right]$, calcium (normal:
2.15-2.58 mmol/l) and creatinine (normal: 0.67-1.4 mg/ $\mathrm{dl}$ for men and 0.56-1.2 mg/dl for women) levels were analyzed, as well as the presence of proteinuria (defined as $>0.2 \mathrm{~g} / 24 \mathrm{~h}$ or positive urinalysis test).

Patients were grouped according to vitamin D status as follows: deficiency - below $20 \mathrm{ng} / \mathrm{ml}$; insufficiency between 20 and $30 \mathrm{ng} / \mathrm{ml}$; optimal levels of $25(\mathrm{OH}) \mathrm{D}_{3}$ - 30 to $100 \mathrm{ng} / \mathrm{ml}$ and potentially toxic concentration above $100 \mathrm{ng} / \mathrm{ml}$. Seasons of blood sampling were categorized as spring (March-May), summer (June-August), autumn (September-November), and winter (DecemberFebruary), in order to assess the potential seasonal variation of vitamin D levels.

Statistical analysis was performed using IBM SPSS Statistics 25 software. A p-value $<0.05$ was considered to indicate statistical significance. Descriptive statistics were calculated for the collected data. The correlations between analyzed parameters were calculated and their significance examined. Distributions of variables were evaluated for normality using the Shapiro-Wilk test. To compare non-parametric distributions for independent variables, the Mann-Whitney U-test and Kruskal-Wallis test were used. If possible, and if the assumptions were met, Student's t-test was used to compare the distribution. In addition, the z-test for the significance of the difference between two structured indicators was used.

The study has a retrospective character; hence the approval of the Bioethical Commission was not required.

\section{Results}

The main clinical characteristics of all 112 participants are presented in Tables I and II. The systemic lupus erythematosus patients were significantly younger than SSc and GPA patients. Women made up the majority of SLE and SSC patients, but less than a half of GPA patients.

Mean serum $25(\mathrm{OH}) \mathrm{D}_{3}$ concentrations were below the cutoff for sufficiency in GPA and SSc patients, and slightly above this value in SLE patients, but they did not significantly differ between the groups $(p=0.54)$. Also when comparing the prevalence of vitamin $D$ deficiency/insufficiency/sufficiency between the diseases (Table III), no significant differences were found ( $p=0.36$ ). Serum concentrations of 25-hydroxyvitamin below the level of $30 \mathrm{ng} / \mathrm{ml}$ were found in $43.8 \%$ of SLE, $65.9 \%$ of SSc and $72.4 \%$ of GPA patients.

$80 \%$ of patients $(81.82 \%$ of SLE, $71.43 \%$ of SSC and $92 \%$ of GPA patients) reported vitamin D intake. The mean daily dose of cholecalciferol was 1398 IU for SLE, 1345 IU for SSC and 1689 IU for GPA.

Regarding other laboratory findings, no statistically significant differences were observed in mean serum calcium levels or in the prevalence of impaired renal 
Table I. Basic clinical characteristics of patients with systemic lupus erythematosus, systemic sclerosis and granulomatosis with polyangiitis

\begin{tabular}{|c|c|c|c|c|c|}
\hline Parameter & $\begin{array}{c}\text { Age } \\
\text { [years] }\end{array}$ & $\begin{array}{c}\mathrm{Ca} \\
{[\mathrm{mmol} / \mathrm{l}]}\end{array}$ & $\begin{array}{l}25(\mathrm{OH}) \mathrm{D}_{3} \\
{[\mathrm{ng} / \mathrm{ml}]}\end{array}$ & $\begin{array}{c}\text { Cholecalciferol dose } \\
\text { [if supplemented] [IU/day] }\end{array}$ & $\begin{array}{l}\text { GC dose } \\
\text { [mg/day] }\end{array}$ \\
\hline \multicolumn{6}{|l|}{$\operatorname{SLE}(n=39)$} \\
\hline Min & 20 & 2.03 & 7.6 & 400 & 0 \\
\hline Max & 73 & 2.51 & 120.8 & 2000 & 36 \\
\hline Mean & 44.05 & 2.3286 & 30.951 & 1398.18 & 11.50 \\
\hline SD & 14.686 & 0.11028 & 19.4073 & 557.311 & 7.443 \\
\hline \multicolumn{6}{|l|}{$\operatorname{SSc}(n=44)$} \\
\hline Min & 32 & 2.18 & 8.0 & 500 & 0 \\
\hline Max & 79 & 2.57 & 72.6 & 6000 & 12 \\
\hline Mean & 56.50 & 2.3815 & 28.847 & 1345.19 & 2.52 \\
\hline SD & 12.155 & 0.10595 & 12.4845 & 743.704 & 3.315 \\
\hline \multicolumn{6}{|c|}{ GPA $(n=29)$} \\
\hline Min & 18 & 1.85 & 8.0 & 400 & 4 \\
\hline Max & 77 & 2.64 & 70.7 & 3000 & 32 \\
\hline Mean & 52.14 & 2.3718 & 28.010 & 1688.70 & 16.93 \\
\hline SD & 14.872 & 0.15630 & 15.2263 & 662.003 & 8.211 \\
\hline
\end{tabular}

$25(\mathrm{OH}) D_{3}-25$-hydroxyvitamin D, GC - glucocorticosteroid (methylprednisolone), GPA - granulomatosis with polyangiitis, SLE - systemic lupus erythematosus, SSc - systemic sclerosis, Max - maximum, Min - minimum, $n$ - number, SD - standard deviation.

Table II. Prevalence of clinical features of patients with systemic lupus erythematosus, systemic sclerosis and granulomatosis with polyangiitis

\begin{tabular}{|lccc|}
\hline Characteristics & SLE & SSc & GPA \\
\hline Female & $36 / 39(92.31 \%)$ & $40 / 44(90.91 \%)$ & $13 / 29(44.83 \%)$ \\
\hline Proteinuria > 0.2 g/24 h & $11 / 38(28.9 \%)$ & $1 / 44(2.3 \%)$ & $8 / 29(27.6 \%)$ \\
\hline Impaired renal function & $15 / 39(38.5 \%)$ & $11 / 44(25.0 \%)$ & $11 / 29(37.9 \%)$ \\
\hline Osteoporosis & $10 / 38(26.3 \%)$ & $7 / 42(16.7 \%)$ & $11 / 28(39.3 \%)$ \\
\hline Bone fractures & $10 / 39(25.6 \%)$ & $4 / 42(9.5 \%)$ & $8 / 28(28.6 \%)$ \\
\hline
\end{tabular}

GPA - granulomatosis with polyangiitis, SLE - systemic lupus erythematosus, SSC-systemic sclerosis.

function (defined as the presence of proteinuria and/or eGFR $<60 \mathrm{ml} / \mathrm{min} / 1.73 \mathrm{~m}^{2}$ ), although proteinuria itself was significantly more common in SLE $(p<0.001)$ and in GPA $(p=0.001)$ than in SSc.

As for the prevalence of osteoporosis and bone fractures in each of the three diseases, we found that they were significantly more frequent in GPA than in SSc (osteoporosis: $39.3 \%$ vs. $16.7 \%, p=0.0341$; bone fractures: $28.6 \%$ vs. $9.5 \%, p=0.0378)$. In systemic lupus erythematosus, osteoporosis and bone fractures were also more common than in SSc, but the differences did not reach statistical significance.

Statistical analysis of the correlations between serum $25(\mathrm{OH}) \mathrm{D}_{3}$ concentrations and selected clinical features was performed for the whole group and for each of the diseases separately. The results are presented in Table IV.
There were no significant correlations between vitamin D levels and age, serum calcium levels, impaired renal function, osteoporosis and history of bone fractures. As for seasonal variations (Table V), observed differences in $25(\mathrm{OH}) \mathrm{D}_{3}$ levels did not reach statistical significance for any of the investigated diseases.

In systemic lupus erythematosus, $25(\mathrm{OH}) \mathrm{D}_{3}$ serum concentration was positively correlated with supplemented vitamin $\mathrm{D}$ dose. In granulomatosis with polyangiitis, serum $25(\mathrm{OH}) \mathrm{D}_{3}$ was significantly higher in women than men. In systemic sclerosis, $25(\mathrm{OH}) \mathrm{D}_{3}$ levels were significantly higher in the group of patients treated with glucocorticosteroids (mean $=33.711$ ) than in the group of patients who did not take steroids (mean $=25.344$ ).

Moreover, in SSc serum $25(\mathrm{OH}) \mathrm{D}_{3}$ was positively correlated with glucocorticosteroid dose. The comparison of glucocorticosteroid-treated vs. non-treated groups 
Table III. Proportions of 25-hydroxyvitamin D $\left[25(\mathrm{OH}) \mathrm{D}_{3}\right]$ levels among patients with systemic lupus erythematosus, systemic sclerosis and granulomatosis with polyangiitis

\begin{tabular}{|c|c|c|c|c|c|c|}
\hline \multirow[t]{2}{*}{$25(\mathrm{OH}) \mathrm{D}_{3}$ level $[\mathrm{ng} / \mathrm{ml}]$} & \multicolumn{2}{|c|}{ SLE } & \multicolumn{2}{|c|}{ SSc } & \multicolumn{2}{|c|}{ GPA } \\
\hline & $n$ & $\%$ & $n$ & $\%$ & $n$ & $\%$ \\
\hline Deficiency $<20$ & 12 & 20.8 & 10 & 22.7 & 8 & 27.6 \\
\hline Insufficiency 20-30 & 9 & 23.0 & 19 & 43.2 & 13 & 44.8 \\
\hline Normal level 30-100 & 17 & 43.6 & 15 & 34.1 & 8 & 27.6 \\
\hline Potentially toxic > 100 & 1 & 2.6 & 0 & 0 & 0 & 0 \\
\hline Total & 39 & 100.0 & 44 & 100.0 & 29 & 100.0 \\
\hline
\end{tabular}

$25(\mathrm{OH}) \mathrm{D}_{3}-25$-hydroxyvitamin D, GPA - granulomatosis with polyangiitis, SLE - systemic lupus erythematosus, SSC - systemic sclerosis.

Table IV. Correlation coefficients between serum $25(\mathrm{OH}) \mathrm{D}_{3}$ concentrations and selected clinical and laboratory variables in patients with systemic lupus erythematosus, systemic sclerosis, granulomatosis with polyangiitis

\begin{tabular}{|lcccccc|}
\hline Parameter & \multicolumn{2}{c}{ SLE } & \multicolumn{2}{c}{ SSc } & \multicolumn{2}{c|}{ GPA } \\
\cline { 2 - 8 } & $r$ & $p$-value & $r$ & $p$-value & $r$ & $p$-value \\
\hline Age & 0.211 & 0.197 & 0.184 & 0.236 & 0.231 & 0.237 \\
\hline Gender & 0.311 & 0.054 & 0.139 & 0.374 & -0.444 & 0.016 \\
\hline Serum calcium level & 0.222 & 0.186 & 0.145 & 0.372 & 0.105 & 0.593 \\
\hline Proteinuria & 0.203 & 0.221 & 0.0 & 0.0 & -0.236 & 0.219 \\
\hline Impaired renal function & 0.207 & 0.206 & 0.265 & 0.086 & -0.329 & 0.81 \\
\hline Osteoporosis & 0.216 & 0.192 & -0.052 & 0.748 & 0.269 & 0.166 \\
\hline Bone fractures & 0.154 & 0.358 & -0.058 & 0.720 & 0.322 & 0.094 \\
\hline Cholecalciferol dose & 0.415 & 0.025 & 0.157 & 0.340 & 0.128 & 0.542 \\
\hline Methylprednisolone dose & 0.106 & 0.539 & 0.342 & 0.029 & -0.147 & 0.446 \\
\hline
\end{tabular}

GPA - granulomatosis with polyangiitis, SLE - systemic lupus erythematosus, SSC - systemic sclerosis.

Table V. Seasonal $25(\mathrm{OH}) \mathrm{D}_{3}$ serum concentrations in patients with systemic lupus erythematosus, granulomatosis with polyangiitis and systemic sclerosis

\begin{tabular}{|c|c|c|c|c|c|}
\hline \multirow[t]{2}{*}{ Disease } & Spring & Summer & Autumn & Winter & \multirow[t]{2}{*}{$p$-value } \\
\hline & \multicolumn{4}{|c|}{$25(\mathrm{OH}) \mathrm{D}_{3}$ mean $(\mathrm{SD})$ concentration $[\mathrm{ng} / \mathrm{ml}]$} & \\
\hline SLE & 26.94 (13.55) & 32.83 (11.11) & $38.01(27.87)$ & 23.57 (16.76) & 0.353 \\
\hline GPA & 33.65 (16.26) & 20.75 (13.36) & 26.51 (12.91) & $28.24(20.41)$ & 0.443 \\
\hline SSc & $30.61(12.29)$ & 35.44 (17.73) & $25.94(7.15)$ & $22.21(9.07)$ & 0.355 \\
\hline
\end{tabular}

$25(\mathrm{OH}) \mathrm{D}_{3}-25$-hydroxyvitamin D, GPA - granulomatosis with polyangiitis, SLE - systemic lupus erythematosus, SSc - systemic sclerosis, p-values represent statistical differences in seasonal $25(\mathrm{OH}) D_{3}$ concentrations.

was not applicable in SLE and GPA, as only a few patients were not treated with glucocorticosteroids.

Of note, among patients with SLE and SSc (but not GPA), there was also a significant positive correlation between osteoporosis prevalence and supplemented vitamin D dose $(r=0.514, p=0.005$ and $r=0.364$, $p=0.019$, respectively).

\section{Discussion}

The results of our study demonstrate high prevalence of low vitamin D levels among patients suffering from different rheumatic diseases, with 71/112 (63.3\%) of them having $25(\mathrm{OH}) \mathrm{D}_{3}$ concentrations below the optimal level of $30 \mathrm{ng} / \mathrm{ml}$ [30]. In particular, $26.8 \%$ of the patients showed $25(\mathrm{OH}) \mathrm{D}_{3}$ deficiency $(<20 \mathrm{ng} / \mathrm{ml})$ and $36.6 \%$ had suboptimal levels of 20 to $30 \mathrm{ng} / \mathrm{ml}$.

However, the epidemiologic data reported by Płudowski et al. [31] demonstrated even higher prevalence of low vitamin D concentrations among the Polish population as compared to our group, with $25(\mathrm{OH}) \mathrm{D}_{3}$ levels below $30 \mathrm{ng} / \mathrm{ml}$ in $89.9 \%$ of studied volunteers. The possible explanation of such differences is that 
the above study was performed during late winter and spring, when the lowest $25(\mathrm{OH}) \mathrm{D}_{3}$ values are expected in the general population due to lack of vitamin D synthesis in the skin, while in our patients vitamin D levels were measured throughout the year, including summer.

In another study based on the Polish adult population, by Kmieć et al. [32], 25(OH)D $\mathrm{D}_{3}$ sufficiency was recorded only in $1.8 \%$ and $14.7 \%$ of participants, during autumn and winter, respectively, which also confirms that the prevalence of decreased vitamin D levels is even higher in the general population than in patients suffering from rheumatic disorders, probably due to the lower frequency of its routine supplementation in healthy subjects.

In our study, we found no significant differences among seasonal $25(\mathrm{OH}) \mathrm{D}_{3}$ concentrations. One likely reason may be that in rheumatic conditions, sunlight exposure is reduced not only by the seasonality, but also by several disease-dependent factors, i.e. reduced mobility, frequent hospitalizations, or avoidance of sunlight exposure in SLE $[17,24]$. On the other hand, the great majority of our patients, especially those on glucocorticosteroid therapy, are routinely supplemented with vitamin D throughout the year as part of osteoporosis prophylaxis, and therefore the seasonal variations of vitamin D status may not be so prominent.

We found the vitamin D intake rate relatively high, reaching $80 \%$ of the studied population, whereas the mean supplemented doses of cholecalciferol fell within the prophylactic dose range (800-2000 IU/day) recommended for adults in Poland [30].

On the other hand, in our study, a significant correlation between $25(\mathrm{OH}) \mathrm{D}_{3}$ level and dose of supplemented vitamin D was found only in the SLE group, whereas in GPA and SSc patients standard vitamin D doses did not significantly influence the $25(\mathrm{OH}) \mathrm{D}_{3}$ status.

Similarly to our results, other studies $[33,34]$ reported that common vitamin D supplementation does not correct the deficiency in SSc patients. One possible explanation may be the reduced vitamin absorption capacity through the thickened intestine wall in the course of the disease [21].

Higher vitamin D levels in SSc patients treated with glucocorticosteroids (which were administered in this order to relieve the symptoms of myositis and/or interstitial lung disease) might be related to potential antiinflammatory GS action within intestines and improved intestinal absorption. Various other obtained results of statistical analysis correlating vitamin D doses and vitamin D serum concentrations may be a consequence of either altered intestinal absorption or inconsistent compliance in some patients.

As for granulomatosis with polyangiitis, given the small number of patients recruited to our study, larger- scale investigations are needed to assess the effect of vitamin $\mathrm{D}$ treatment on correcting $25(\mathrm{OH}) \mathrm{D}_{3}$ deficiency.

Interestingly, we found no statistically significant differences in $25(\mathrm{OH}) \mathrm{D}_{3}$ levels between the SLE, GPA and SSc patients, despite different prevalence of factors known to be associated with vitamin D deficiencies, i.e. osteoporosis, bone fractures and proteinuria [17, 35-38].

Additionally, although the prevalence of $25(\mathrm{OH}) \mathrm{D}_{3}$ insufficiency/deficiency varied between the diseases (43.8\% in SLE, $65.9 \%$ in SSc and $72.4 \%$ in GPA), the differences did not reach statistical significance $(p=0.266)$.

One possible explanation of the similar frequency of low vitamin $\mathrm{D}$ levels among patients with different rheumatic disorders may be that there are other common determinants, such as geographical latitude, nutritional habits, lifestyle or the chronic nature of the disease itself, that play a crucial role in maintaining vitamin D status, irrespectively of the diagnosis.

On the other hand, the lack of statistically significant differences may be due to the too small size of the investigated subgroups, and further studies on larger cohorts are planned to confirm and expand these results showing e.g. the percentage of patients with $25(\mathrm{OH}) \mathrm{D}_{3}$ levels $>30 \mathrm{ng} / \mathrm{ml}$ according to the dosage of vitamin $\mathrm{D}$ and selected clinical parameters.

To date, no specific guidelines regarding dosing of vitamin D in Polish patients with rheumatic diseases have been clearly established. However, taking into account the prevalence of vitamin D insufficiency and deficiency in our group, despite its common supplementation, it seems that patients suffering from rheumatic diseases should be recommended to take vitamin D at higher doses than the general population, preferentially adjusted to serum $25(\mathrm{OH}) \mathrm{D}_{3}$ concentration.

We suggest individualizing the dosage in these patients, starting with 2000 IU of cholecalciferol daily, followed by serum level assessment after 3 months and subsequent appropriate dosage modifications to maintain concentrations within the optimal range.

\section{Conclusions}

We have found that the problem of vitamin D insufficiency and deficiency is quite common among patients with rheumatic disorders, despite high frequency of vitamin D supplementation. To date, no recommendations regarding vitamin D supplementary doses have been established for this specific group of patients in Poland.

However, in view of the ongoing studies regarding potential associations between vitamin D concentrations and prevalence and outcomes of rheumatic diseases, it seems reasonable to advise its regular prophylactic administration. 
The present results suggest that the cholecalciferol dosage recommended for the general population is not sufficient for patients with rheumatic diseases and that seasonal changes of doses are not advisable in these diseases. The present study supports the use of higher initial daily doses of vitamin D (2000 up to 4000 UI) for patients with rheumatic diseases.

Further research in a large group of patients taking various doses of vitamin D should make it possible to establish the appropriate dosage required in selected rheumatic diseases.

The authors declare no conflict of interest.

\section{References}

1. Murdaca G, Tonacci A, Negrini S, et al. Autoimmunity reviews emerging role of vitamin $D$ in autoimmune diseases: an update on evidence and therapeutic implications. Autoimmun Rev 2019; 18: 102350, DOI: 10.1016/j.autrev.2019.102350.

2. Stumpf WE, Sar M, Reid FA, et al. Target cells for 1,25-dihydroxyvitamin $\mathrm{D}_{3}$ in intestinal tract, stomach, kidney, skin, pituitary, and parathyroid. Science 1979; 206: 1188-1190, DOI: $10.1126 /$ science. 505004 .

3. Grygiel-Górniak B, Puszczewicz M. Vitamin D - a new look in medicine and rheumatology. Postepy Hig Med Dosw (Online) 2014; 68: 359-368, DOI: 10.5604/17322693.1097426 [Article in Polish].

4. Myszka M, Klinger $M$. The immunomodulatory role of vitamin D. Postepy Hig Med Dosw (Online) 2014; 68: 865-878, DOI: 10.5604/17322693.1110168 [Article in Polish].

5. Saponaro F, Marcocci C, Zucchi R. Vitamin D status and cardiovascular outcome. J Endocrinol Invest 2019; 42: 1285-1290, DOI: 10.1007/s40618-019-01057-y.

6. Pittas AG, Lau J, Hu FB, Dawson-Hughes B. Review: the role of vitamin $D$ and calcium in type 2 diabetes. A systematic review and meta-analysis. J Clin Endocrinol Metab 2007; 92: 20172029, DOI: 10.1210/jc.2007-0298.

7. Lappe JM, Travers-Gustafson D, Davies KM, et al. Vitamin D and calcium supplementation reduces cancer risk: results of a randomized trial. Am J Clin Nutr 2008; 87: 794-794, DOI: 10.1093/ajcn/87.3.794 [Erratum].

8. Yamshchikov AV, Desai NS, Blumberg HM, et al. Vitamin D for treatment and prevention of infectious diseases: a systematic review of randomized controlled trials. Endocr Pract 2009; 15: 438-449, DOI: 10.4158/EP09101.ORR.

9. Adorini L, Penna G. Control of autoimmune diseases by the vitamin D endocrine system. Nat Clin Pract Rheumatol 2008; 4: 404-412, DOI: 10.1038/ncprheum0855.

10. Theodoratou E, Tzoulaki I, Zgaga L, loannidis J. Vitamin D and multiple health outcomes: umbrella review of systematic reviews and meta-analyses of observational studies and randomised trials. BMJ 2014; 2035: 1-19, DOI: 10.1136/bmj.g2035.

11. Abrahamsen B, Harvey NC. The role of vitamin D supplementation in patients with rheumatic diseases. Nat Rev Rheumatol 2013; 9: 411-422, DOI: 10.1038/nrrheum.2013.71.
12. Buckley L, Guyatt G, Fink HA, et al. 2017 American College of Rheumatology guideline for the prevention and treatment of glucocorticoid-induced osteoporosis. Arthritis Rheumatol 2017; 69: 1521-1537, DOI: 10.1002/art.40137.

13. Duru N, van der Goes MC, Jacobs JW, et al. EULAR evidencebased and consensus-based recommendations on the management of medium to high-dose glucocorticoid therapy in rheumatic diseases. Ann Rheum Dis 2013; 72: 1905-1913, DOI: 10.1136/annrheumdis-2013-203249.

14. Wang XR, Xiao JP, Zhang JJ, Wu YG. Decreased serum/plasma vitamin D levels in SLE patients: a meta-analysis. Curr Pharm Des 2018; 24: 4466-4473, DOI: 10.2174/1381612825666190111 145848.

15. Adami G, Rossini M, Bogliolo L, et al. An exploratory study on the role of vitamin $D$ supplementation in improving pain and disease activity in rheumatoid arthritis. Mod Rheumatol 2019; 29: 1059-1062, DOI: 10.1080/14397595.2018.1532622.

16. Lin J, Liu J, Davies ML, Chen W. Serum vitamin D level and rheumatoid arthritis disease activity: review and meta-analysis. PLoS One 2016; 11: e0146351, DOI: 10.1371/journal.pone. 0146351.

17. Amital H, Szekanecz Z, Szücs G, et al. Serum concentrations of $25-\mathrm{OH}$ vitamin $\mathrm{D}$ in patients with systemic lupus erythematosus (SLE) are inversely related to disease activity: is it time to routinely supplement patients with SLE with vitamin D? Ann Rheum Dis 2010; 69: 1155-1157, DOI: 10.1136/ard.2009.120329.

18. Kerr GS, Sabahi I, Richards JS, et al. Prevalence of vitamin D insufficiency/deficiency in rheumatoid arthritis and associations with disease severity and activity. J Rheumatol 2011; 38: 53-59, DOI: 10.3899/jrheum.100516.

19. Rossini M, Bongi SM, La Montagna G, et al. Vitamin D deficiency in rheumatoid arthritis: prevalence, determinants and associations with disease activity and disability. Arthritis Res Ther 2010; 12: R216, DOI: 10.1186/ar3195.

20. Guan SY, Cai HY, Wang P, et al. Association between circulating 25-hydroxyvitamin $D$ and systemic lupus erythematosus: a systematic review and meta-analysis. Int J Rheum Dis 2019; 22: 1803-1813, DOI: 10.1111/1756-185X.13676.

21. An L, Sun MH, Chen F, Li JR. Vitamin D levels in systemic sclerosis patients: a meta-analysis. Drug Des Devel Ther 2017; 11: 3119-3125, DOI: 10.2147/DDDT.S144860.

22. Giuggioli D, Colaci M, Cassone G, et al. Serum $25-\mathrm{OH}$ vitamin D levels in systemic sclerosis: analysis of 140 patients and review of the literature. Clin Rheumatol 2017; 36: 583-590, DOI: 10.1007/s10067-016-3535-z.

23. Arnson Y, Amital H, Agmon-Levin N, et al. Serum $25-\mathrm{OH}$ vitamin $D$ concentrations are linked with various clinical aspects in patients with systemic sclerosis: a retrospective cohort study and review of the literature. Autoimmun Rev 2011; 10: 490-494, DOI: 10.1016/j.autrev.2011.02.002.

24. Trombetta AC, Smith V, Gotelli E, et al. Vitamin D deficiency and clinical correlations in systemic sclerosis patients: a retrospective analysis for possible future developments. PLoS One 2017; 12: e0179062, DOI: 10.1371/journal.pone.0179062.

25. Caimmi C, Bertoldo E, Pozza A, et al. Vitamin D serum levels and the risk of digital ulcers in systemic sclerosis: a longitudinal study. Int J Rheum Dis 2019; 22: 1041-1045, DOI: 10.1111/1756-185X.13554. 
26. Perez MO, Oliveira RM, Levy-Neto M, et al. Serum 25-hydroxyvitamin $D$ levels in patients with granulomatosis with polyangiitis: association with respiratory infection. Clinics (Sao Paulo) 2017; 72: 723-728, DOI: 10.6061/clinics/2017(12)02.

27. Hochberg MC. Updating the American College of Rheumatology revised criteria for the classification of systemic lupus erythematosus. Arthritis Rheum 1997; 40: 1725-1725, DOI: 10.1002/art.1780400928.

28. van den Hoogen F, Khanna D, Fransen J, et al. 2013 classification criteria for systemic sclerosis: an American College of Rheumatology/European League Against Rheumatism collaborative initiative. Ann Rheum Dis 2013; 72: 1747-1755, DOI: 10.1136/annrheumdis-2013-204424.

29. Leavitt RY, Fauci AS, Bloch DA, et al. The American College of Rheumatology 1990 criteria for the classification of Wegener's granulomatosis. Arthritis Rheum 2010; 33: 1101-1107, DOI: 10.1002/art.1780330807.

30. Rusińska A, Płudowski P, Walczak M, et al. Vitamin D supplementation guidelines for general population and groups at risk of vitamin D deficiency in Poland - recommendations of the Polish Society of Pediatric Endocrinology and Diabetes and the Expert Panel With Participation of National Specialist C. Front Endocrinol (Lausanne) 2018; 9: 246, DOI: 10.3389/fendo. 2018.00246

31. Płudowski P, Ducki C, Konstantynowicz J, Jaworski M. Vitamin D status in Poland. Pol Arch Med Wewn 2016; 126: 530-539, DOI: 10.20452/pamw.3479.
32. Kmieć P, Zmijewski M, Lizakowska-Kmieć M, Sworczak K. Widespread vitamin $D$ deficiency among adults from northern Poland $\left(54^{\circ} \mathrm{N}\right)$ after months of low and high natural UVB radiation. Endokrynol Pol 2015; 66: 30-38, DOI: 10.5603/EP. 2015.0006.

33. Vacca A, Cormier C, Piras M, et al. Vitamin D deficiency and insufficiency in 2 independent cohorts of patients with systemic sclerosis. J Rheumatol 2009; 36: 1924-1929, DOI: 10.3899/jrheum. 081287.

34. Groseanu L, Bojinca V, Gudu T, et al. Low vitamin D status in systemic sclerosis and the impact on disease phenotype. Eur J Rheumatol 2016; 3: 50-55, DOI: 10.5152/eurjrheum.2015.0065.

35. Aggarwal A, Yadav AK, Ramachandran R, et al. Bioavailable vitamin $D$ levels are reduced and correlate with bone mineral density and markers of mineral metabolism in adults with nephrotic syndrome. Nephrology 2016; 21: 483-489, DOI: 10.1111/nep.12638.

36. Banerjee $S$, Basu $S$, Akhtar $S$, et al. Free vitamin D levels in steroid-sensitive nephrotic syndrome and healthy controls. Pediatr Nephrol 2020; 35: 447-454, DOI: 10.1007/s00467-01904433-1.

37. Chapuy MC, Arlot ME, Duboeuf F, et al. Vitamin $D_{3}$ and calcium to prevent hip fractures in elderly women. N Engl J Med 1992; 327: 1637-1642, DOI: 10.1056/NEJM199212033272305.

38. Dawson-Hughes B, Harris SS, Krall EA, Dallal GE. Effect of calcium and vitamin $D$ supplementation on bone density in men and women 65 years of age or older. N Engl J Med 1997; 337: 670-676, DOI: 10.1056/NEJM199709043371003. 